\section{Meet the contributors}

\author{
Nicola Oberbeckmann-Winter ${ }^{1}$ (D)
}

Published online: 7 August 2020

(C) Springer-Verlag GmbH Germany, part of Springer Nature 2020

This special collection published in $\mathrm{ABC}$ features female role models in (bio-)analytical chemistry. Exceptional female researchers were recommended to us by the journal's International Advisory Board. The authors below accepted our invitation and submitted outstanding work that was considered by referees and the $\mathrm{ABC}$ Editors as being of high interest for our readers. They represent a geographically diverse - with almost 20 countries represented - overview over a generation of upcoming and renowned experts who inspire students and postdocs.

We express gratitude for the positive feedback we have received, thank all authors for their various contributions, and invite you to browse through this presentation of the authors in addition to their publications within this collection.

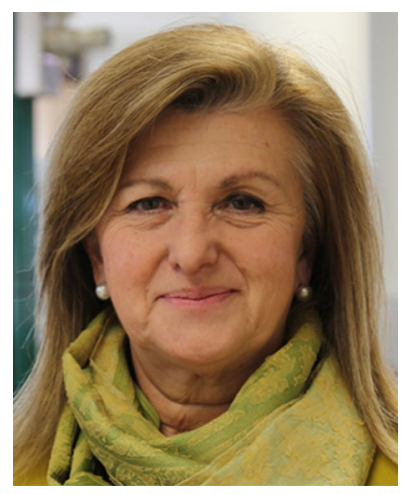

Coral Barbas is Head of the Centre for Metabolomics and Bioanalysis (CEMBIO) at San Pablo CEU University in Madrid. She is also Director for the CEU International School of Doctorate. She was awarded with a honoris causa Doctorate

Published in the topical collection featuring Female Role Models in Analytical Chemistry.

Nicola Oberbeckmann-Winter

nicola.oberbeckmann-winter@ springer.com

1 Analytical and Bioanalytical Chemistry, Springer, Tiergartenstrasse 17, 69121 Heidelberg, Germany by Bialystok Medical University in 2018 and with the prize for the Belgian Society of Pharmaceutical Sciences. She has been included in the power list of Analytical Scientist for several years. Among her current research interests are all aspects of the analytical workflow in metabolomics with mass spectrometry from sample collection to metabolite identification.

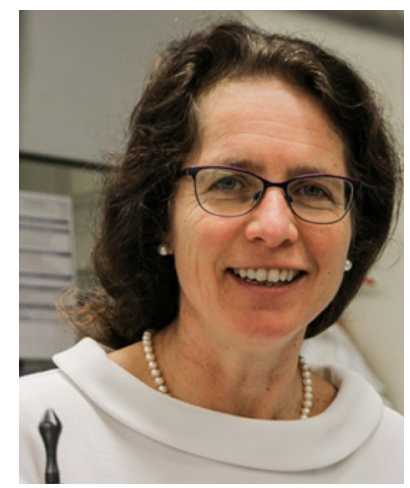

Antje Baeumner is Director of the Institute of Analytical Chemistry, Chemo- and Biosensors at the University of Regensburg. Prior to returning to Germany, she was Professor and Director of Graduate Studies in the Department of Biological and Environmental Engineering at Cornell University in Ithaca, NY, USA. She is Editor of the Springer Nature Journal Analytical and Bioanalytical Chemistry and President of the International Association of Environmental Analytical Chemistry (IAEAC). Her research is focused on the development of biosensors and micrototal analysis systems for the detection of pathogens and toxins in food, the environment and for clinical diagnostics. Her research includes the development of novel nanomaterials, such as liposomes, nanofibers, and nanoparticles; microfluidic biosensors; sample preparation strategies; and point-of-care devices. She chaired the Gordon Research Conference (GRC) on Bioanalytical Sensors in 2010 and is co-chair for the GRC on Nanoscale Science and Engineering for Agricultural and Food Systems in 2020. She also organized and chaired the international biosensor conference BBMEC in 2001 and 2015. She has received numerous 
awards for her research, including being a finalist of the Blavatnik Award, senior fellow of the Alexander-vonHumboldt Foundation, Mercator Professor of the German Science Foundation, and numerous teaching awards.

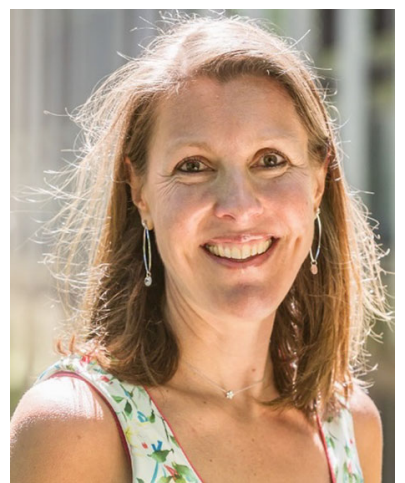

Annemie Bogaerts is Full Professor at the University of Antwerp, Department of Chemistry, and head of the research group PLASMANT (Plasma Lab for Applications in Sustainability and Medicine - ANTwerp). Her research focuses on the study of non-equilibrium plasmas, by modelling the plasma chemistry, plasma reactor design and plasma-surface interactions, as well as by experiments. Initially, she focused on plasmas for analytical chemistry, for which she obtained the Winter Plasma Award (2009) and the Lester W. Strock Award of the Society for Applied Spectroscopy (2008). Current applications of her research are in sustainable chemistry and energy/environmental applications (e.g., greenhouse gas conversion) and in plasma for cancer treatment). She has an ERC Synergy Grant on plasma catalysis for electrification of chemical reactions (SCOPE), together with G. Centi, V. Hessel and E. Rebrov.

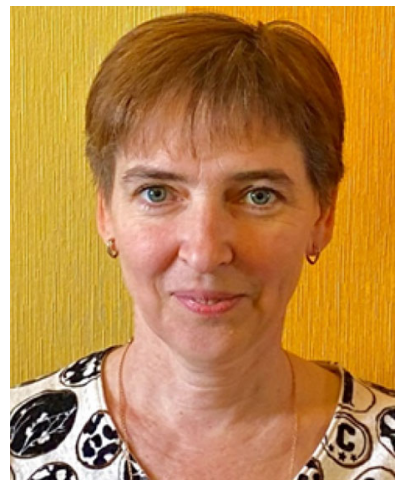

Natalia Burmistrova is Professor of the Department of General and Inorganic Chemistry at the Institute of Chemistry Saratov State University, Russia. Her main research interest is chemical sensing based on microstructured optical fibers, as well as developing dyes and luminescent probes serve to render biomolecules (e.g., hydrogen peroxide, horseradish peroxidase) amenable for quantitation with optical readout.

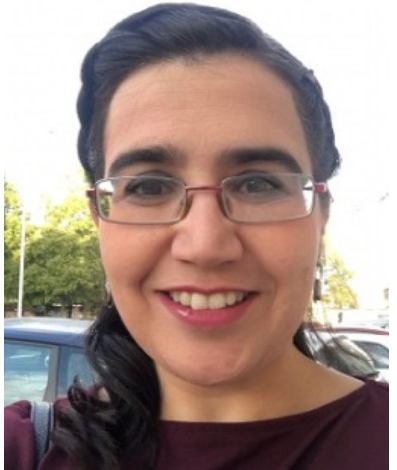

Susana Campuzano works as Assistant Professor at the Analytical Chemistry Department of the Chemistry Faculty of the Universidad Complutense de Madrid, where she belongs to the Electroanalysis and Electrochemical (Bio)sensors research group. Her current research lines focus on the development of electroanalytical bioplatforms for individual or multiplexed determination of biomarkers at different molecular levels with practical applicability in the food or clinical fields. She has been Assistant Editor of the journal Electroanalysis since January 2019.

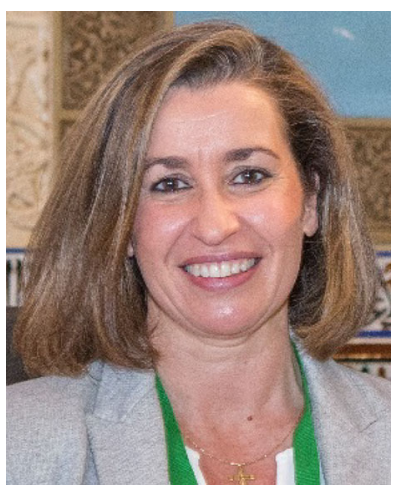

Soledad Cárdenas Aranzana is Full Professor of Analytical Chemistry and head of the research group FQM215 from the Department of Analytical Chemistry, University of Córdoba. Her research interests comprise microextraction techniques, including the development of new approaches and the synthesis of novel nanomaterials based on carbon, metallic nanoparticles, and polymers.

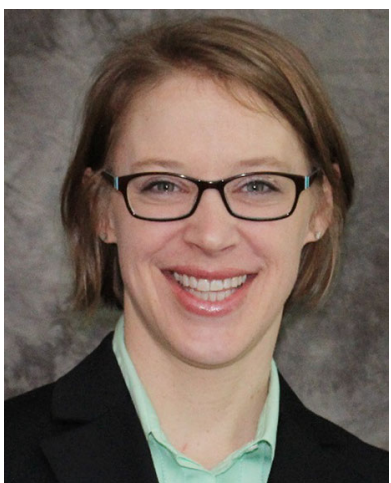

Julie Champion is Associate Professor in the School of Chemical and Biomolecular Engineering at Georgia 
Institute of Technology. She received a BRIGE award from the National Science Foundation, the Georgia Tech Women in Engineering Faculty Award for Excellence in Teaching, and the Georgia Tech BioEngineering Program Outstanding Advisor Award. Her current research focuses on the design and selfassembly of functional materials made from engineered proteins for applications in immunology; cancer; and, more recently, biocatalysis.

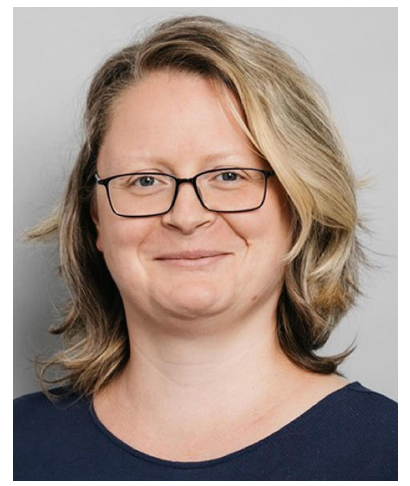

Dana Cialla-May is Group Leader at the Leibniz Institute of Photonic Technology, in Jena, Germany, within the Department of Spectroscopy/Imaging. She received her $\mathrm{PhD}$ in 2010 from the Friedrich-Schiller University, Jena, Germany, under the supervision of Prof. Dr. Jürgen Popp. Her research focuses on surfaceenhanced Raman spectroscopy (SERS) in bioanalytical detection schemes, including drug monitoring and analysis in complex biological matrices.

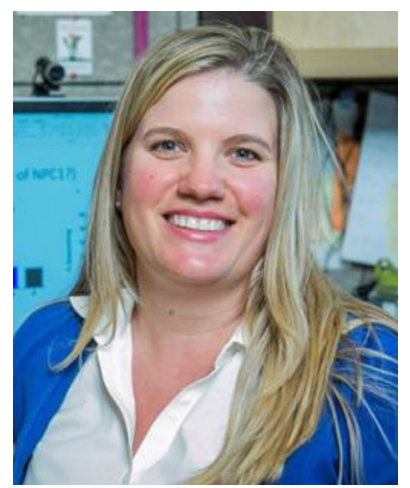

Susan Daniel is Associate Professor in the RF Smith School of Chemical and Biomolecular Engineering at Cornell University. Her research interests are in understanding phenomena at biological interfaces and chemically patterned surfaces that interact with soft matter, namely, liquids; polymers; and biological materials, such as cells, viruses, proteins, and lipids. She leads a biotechnology research group that pioneered the use of "biomembrane chips" to conduct cellfree, membrane biophysical studies of mammalian, bacterial, and plant cell membranes, and recently merged this technology with organic electronic devices for expanded sensing capabilities. She is the recipient of a National Science Foundation CAREER award (2011); the Denice Denton Emerging Leader Award (2012); the Schwartz Life Sciences award (2016); and, in 2017, she was honored with the College of Engineering's Research Excellence Award at Cornell University.

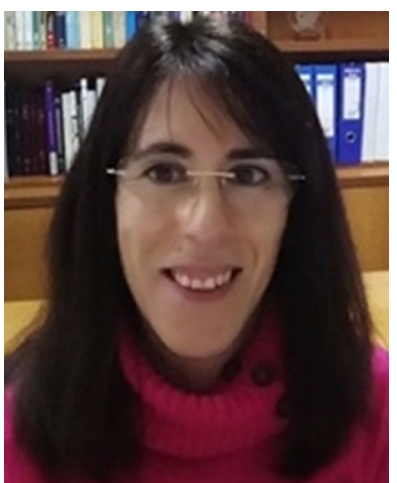

Noemí de-los-Santos-Álvarez is Associate Professor of Analytical Chemistry at the University of Oviedo (Spain). Her current research interests include the selection and characterization of aptamers and the development of aptasensors and genosensors for tumor biomarker detection. She has taught analytical chemistry at undergraduate level in chemistry, chemical engineering and biotechnology degrees and also at graduate level in chemistry, where she is implementing several teaching innovation projects aimed at actively involving students in the classroom.

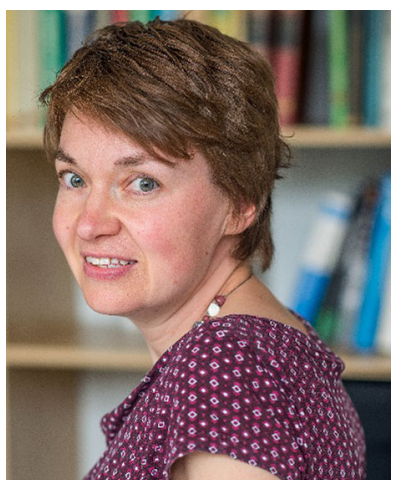

Karolien De Wael has a wide expertise in (bio)electrochemistry and is spokesperson of the AXES Research Group at the University of Antwerp in Belgium. She is co-chair of the Enviromics Consortium (Industrial Research Fund) and co-promotor of the Nanolab Center of Excellence within the university. Her strategic vision aims at a portfolio of sensor technologies that can be applied in different markets/sectors, embracing the idea of responsible research and innovation. 


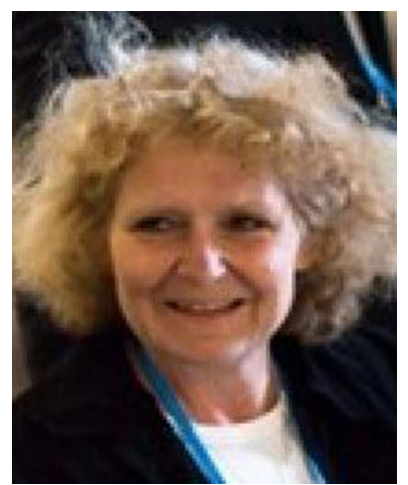

Jenny Emnéus heads the Bioanalytics group at DTU Bioengineering. She has pioneered work on neural stem cell differentiation on carbon-based optoelectrical waveguides, with optogenetic control of dopamine release from dopaminergic neurons. She was one of the first to realize chip-based microfluidic immunoassays and the first to develop the immuno affinity based SLM extraction technique. Her research interests include electrochemical 2D/3D (bio)sensing/(bio)sensor arrays (voltammetry and impedance), polymer microfabrication and 3D printing of LOCs and 3D scaffolds for bioanalysis and tissue engineering/bioartificial organs, and microfluidic/perfusion-based cell and tissue culture systems. She has coordinated numerous EU projects and is very active in research evaluation, serving eight years as a panel member of the European Research Council.

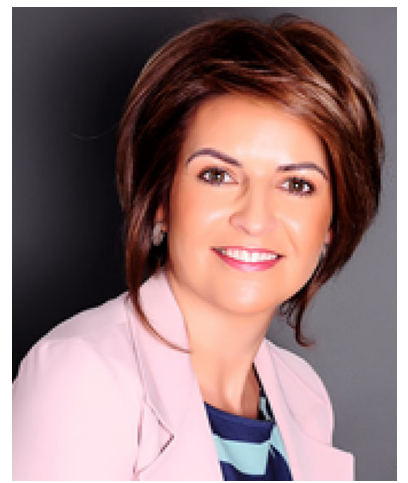

Márcia Foster Mesko is Associate Professor at the Federal University of Pelotas (UFPel)/Brazil (since 2009) and she is Head of the Laboratory of Control of Contaminants in Biomaterials. Currently, she is Director of the Analytical Chemistry Division of the Brazilian Chemical Society, and coordinator of the Biochemistry and Bioprospecting Postgraduate Program at UFPel. She has received several national and international awards, including the "L'Oréal Brazil - For Woman in Science" from L'Oréal, Brazilian Academy of Science and UNESCO, and the "Periodic Table of Younger Chemists" to represent the element "Bromine" for celebrating 100 years of IUPAC and the International Year of Periodic Table. Recently, she was elected as affiliate member of the Brazilian Academy of Science Chemistry Sciences. She has published 100 peerreviewed international papers and more than 15 book chapters. She has experience in the development of methods, especially for halogen determination, using atomic spectrometry and chromatography techniques.

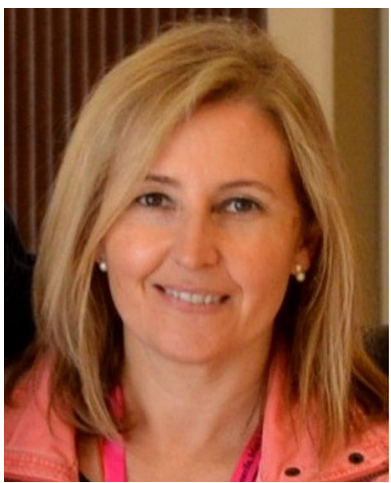

Ana María Garcia-Campaña is Full Professor and Head of the Department of Analytical Chemistry at the University of Granada (Spain). Currently, she is President of the Spanish Society of Chromatography and Related Techniques (SECyTA). She has the main responsibility for the research group "Quality in Food, Environmental and Clinical Analytical Chemistry," and her research fields are related to LC and CE coupled with UV-Vis, luminescence and MS, using innovative sample treatments in accordance with green chemistry principles, mainly focused on food safety and quality control, drug monitoring in biological fluids and determination of contaminants and residues (pesticides, veterinary drugs, and natural toxins) in foods, herbal products, nutraceuticals, and environmental samples.

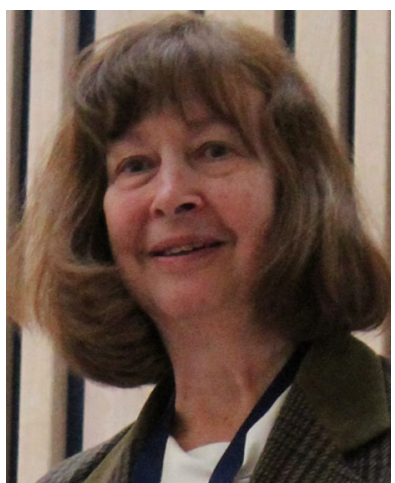

Elizabeth (Lisa) A. H. Hall leads Cambridge Analytical Biotechnology. Her research focusses on understanding how biology can be interfaced with electronic, mechanical and optical systems and new ways to answer fundamental and applied questions concerning new 
measurement regimes. This research links transduction technologies with synthetic biology and nanomaterials. Her work has been awarded with the Gold Medal from the Royal Society of Chemistry, Analytical Division (2005); the Alec Hough-Grassby Award (2009); the SNU-Dongjin Distinguished Lectureship (2019) and the Oxburgh Medal (2020). She is a Liveryman of the Worshipful Company of Instrument Makers and she was made Commander of the Most Excellent Order of the British Empire (CBE) in the Queen's Birthday Honours List 2015 for services to Higher Education and Sport for Disabled.

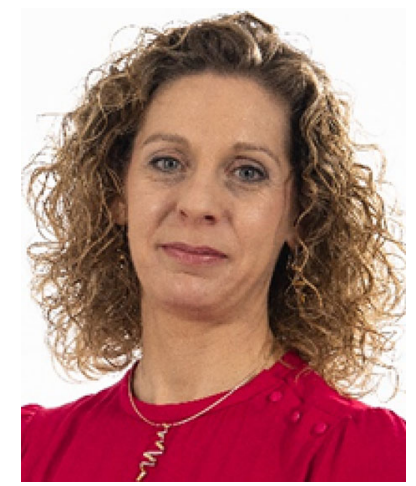

Aranzazu Heras received her BS and her PhD in chemistry from the Universidad de Burgos (Spain), where she has been working as Associate Professor in Analytical Chemistry since 1997. Her research interests include different aspects related to SEC (UV-Vis-NIR, photoluminescence, Raman), from design and development of new devices to applications of this technique in analysis, characterization of organic, inorganic and nanomaterials, and in the study of different reaction mechanisms. Her research group, Instrumental Analysis Group, at the University of Burgos was recognized with the "Scientific research" CIDETEC award 2011-2014 related to advances carried out in spectroelectrochemistry and the transfer of know-how.

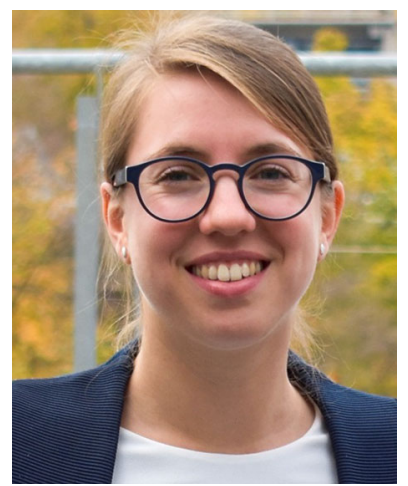

Cornelia A. Hermann is a chemistry postdoctoral researcher in the research group of Prof. Antje J. Baeumner at the Institute of Analytical Chemistry, Chemo- and Biosensors of the University of Regensburg in Germany, where she finished her Ph.D. studies in 2019. Her research focuses on the use of liposomes in DNA hybridization assays, their modification with magnetic properties and application in microfluidic systems.

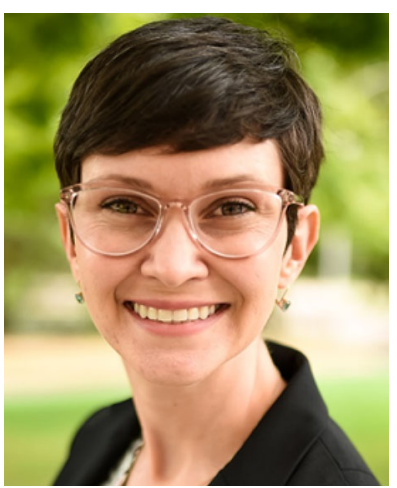

Kelly Hines is Assistant Professor of Chemistry at the University of Georgia. The Hines Lab develops and applies bioanalytical methods using ion mobility-mass spectrometry (IM-MS) to enhance the throughput and dimensionality of lipidomics and metabolomics experiments. Applications of these approaches include the characterization of metabolic alterations in antibiotic resistant pathogens and the development of IM-MS methods for antibiotic susceptibility testing and small-molecule screening.

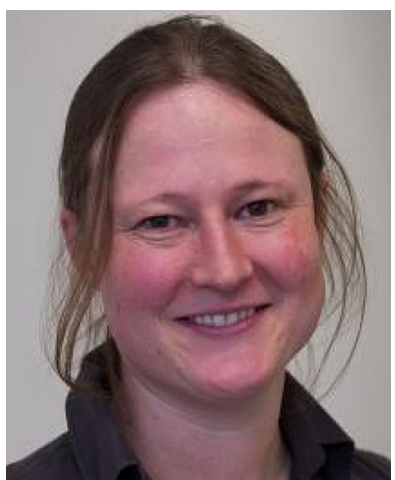

Carolin Huhn is a chemist by training and Professor for Effect-Based Environmental Analysis at the Eberhard Karls Universität Tübingen. Since 2013, she has been the speaker of the platform Environmental System Analysis at the university. Her research interests cover all aspects of analytical chemistry: instrumental development, analytical basic research, method development, and applications. Her main focus is on electromigrative separation techniques, including multidimensional separations and hyphenation to mass spectrometry. New methods are applied in various fields such as environmental (bio-)analysis, forensics, clinical chemistry and pharmacy with cooperation partners. 


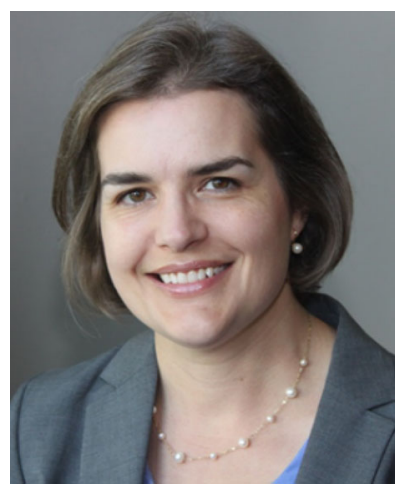

Amanda Hummon is Associate Professor of Chemistry and Biochemistry at The Ohio State University and a member of Ohio State's Comprehensive Cancer Center - Arthur G. James Cancer Hospital and Richard J. Solove Research Institute. Her research group examines protein changes that occur with the development and progression of colorectal cancer and they also develop novel imaging mass spectrometry approaches. She has been recognized with an NSF CAREER award, an SACP Starter grant, an ACS Rising Star Award, and a Presidential Early Career Award for Scientists and Engineers (PECASE).

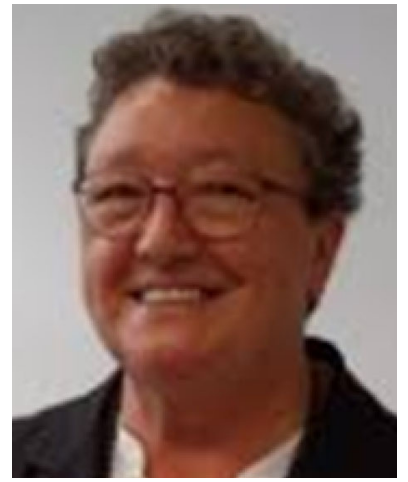

Nicole Jaffrezic-Renault received her engineering degree from Chimie ParisTech, in 1971, and her Doctorat d'Etat és Sciences Physiques from the University of Paris in 1976. She is Emeritus Director of Research at the Centre National de la Recherche Scientifique, past President of the chemical micro sensor club (CMC2), President of the Analytical Division, and member of the administrative council of the French Chemical Society. She was decorated with Knight of the Legion of Honor and Officer of the National Order of Merit. Her research activities in the Institute of Analytical Sciences include conception and design of (bio)chemical sensors and their integration in microsystems. She coordinates several European and national projects for the development of microsystems for biomedical and environmental monitoring and for food safety. She has published more than 560 papers with more than 11,400 citations (h index 53).

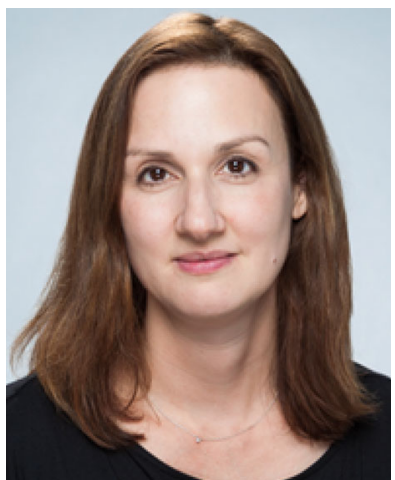

Catherine Klapperich is Founding Director of the Precision Diagnostics Center at Boston University. She is Professor and Vice Chair of Biomedical Engineering. Her research is focused on engineering lab-on-a-chip diagnostic tests for use in low-resource settings and at the point of care. She works in the areas of women's health, infectious disease diagnostics, drug adherence, and continuous physiological monitoring.

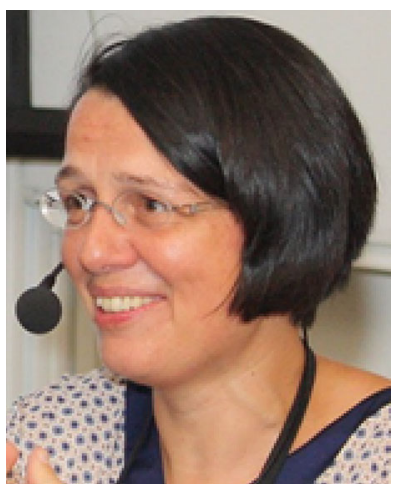

Janina Kneipp is Professor of Physical Chemistry at Humboldt-Universität zu Berlin (Germany). She works in the field of optical nanospectroscopy. Her work combines concepts of modern developments in vibrational microscopy, nanotechnology, and plasmonics, with the overall objective of laying groundwork for applications in analytical and biophysical chemistry. The chemical characterization and imaging of complex biological samples, such as cells and tissues from animals and plants, have been in the focus of her research for the past 20 years. She was the 2019 Caroline von Humboldt Professor, has been a Wilhelm Ostwald Fellow of BAM Federal Institute for Materials Research and Testing since 2012, and received the Bunsen Kirchhoff Award of the German Chemical Society, as well as an ERC Starting Grant in 2010 . 


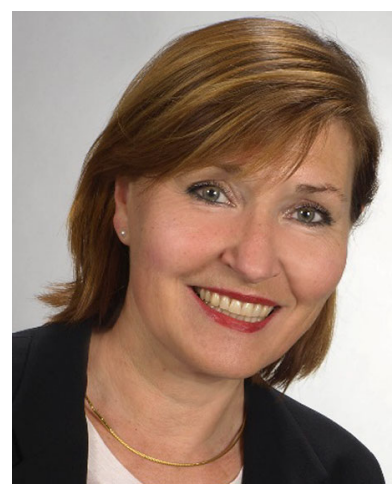

Christine Kranz is Head of the surface science group at the Institute of Analytical and Bioanalytical Chemistry, Ulm University. She is currently co-speaker of the DFG-funded Research Training Group PULMOSENS, which is focused on innovative sensing approaches in lung research. Her main research interest is in field scanning probe microscopy and miniaturized amperometric biosensors for life science applications.

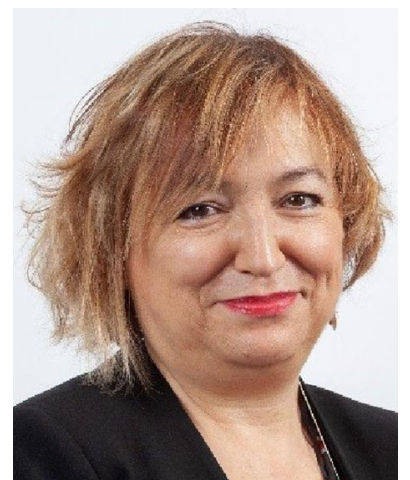

Laura M. Lechuga is Full Professor of the Spanish National Research Council (CSIC) and Head of the Nanobiosensors and Bioanalytical Applications Group at the Catalan Institute of Nanoscience and Nanotechnology (ICN2) in Barcelona (Spain) and at the Networking Biomedical Research Center (CIBER-BBN). The principal focus of her research is the development of novel nanobiosensor devices based on nanoplasmonics and silicon-based photonics principles, including surface biofunctionalization, microfluidics for automatic fluid delivery and complete lab-on-a-chip integration for point-of-care devices.

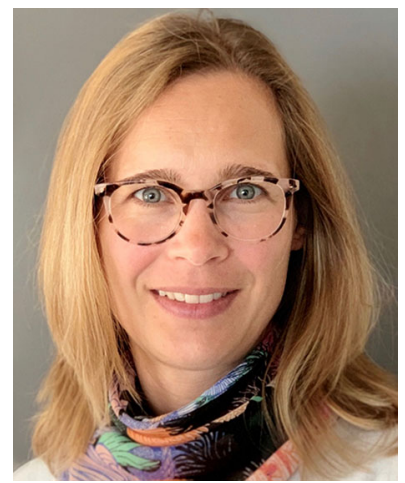

Kerstin Leopold is Professor of Analytical Chemistry and Deputy Director of the Institute of Analytical and Bioanalytical Chemistry (IABC) at Ulm University, Germany. Her research field is the development of new methodologies in trace and ultratrace analysis, speciation, and nanoanalysis. Thereby, various atomic spectrometry techniques, such as AFS, AAS, AES, TXRF, and $\mu \mathrm{XRF}$, are applied and further developed. Moreover, projects strive for application to real-world samples coming from environmental, biological, and medical research. In 2015, she received the Mileva Einstein-Marić-Award given by Ulm University in recognition of her outstanding research work, dedicated teaching, and ideal balance of academic career and family responsibilities.

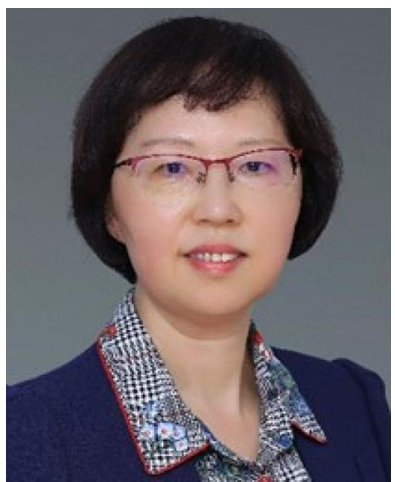

Yao-Qun Li (PhD) is Full Professor in Chemistry at Xiamen University, China. Her current research interests include fluorescence spectroscopy, surface plasmons, optical imaging, biomimetic nanopore techniques, and rapid detection for food safety and bioassay. She received the Science and Technology Progress Award from the Ministry of Education, China.

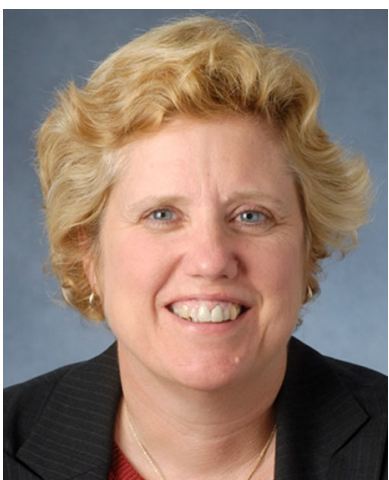

Susan M. Lunte is the Ralph N. Adams Distinguished Professor of Chemistry and Pharmaceutical Chemistry, Director of the Adams Institute for Bioanalytical Chemistry, and Director of the NIH COBRE Center for Molecular Analysis of Disease Pathways at the University of Kansas, Lawrence, KS. Her research interests include the development of new methodologies for the separation and detection of peptides, amino acids, 
neurotransmitters, and pharmaceuticals in biological fluids. This includes separation-based sensors for the continuous monitoring of drugs and neurotransmitters in freely roaming animals and new methodologies for the determination of reactive oxygen and nitrogen species in cells.

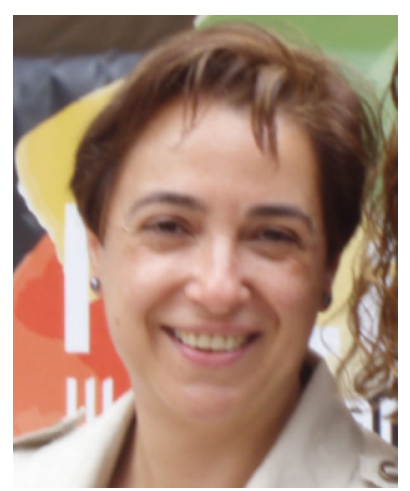

Yolanda Madrid is Full Professor in Analytical Chemistry in the Department of Analytical Chemistry, Faculty of Chemistry, Complutense University, Madrid, Spain. She studied chemistry at the Complutense University of Madrid, where she defended her doctoral thesis in February 1991 in the Group of Prof. Carmen Camara. In September 1991, she joined the group of Prof. Gary M. Hieftje at the Indiana University, Bloomington, IN (USA) as Postdoctoral Fellow until October 1992. Her key expertise lies in trace-element speciation, metallomics research, and the combined used of elemental and molecular mass spectrometry. Nowadays, her research topics are also focused on the synthesis, characterization, and quantification of metallic nanoparticles and metallic oxide nanoparticles in complex matrices (environmental, biological, food, and consumer products). She is co-author of more than 100 scientific publications and reviews ( $h$ index 38). She has participated in different research projects (national, regional, and international) through which the research is funded. She belongs to the Atomic Spectrometry Updates editorial board of the Journal of Analytical Atomic Spectrometry.

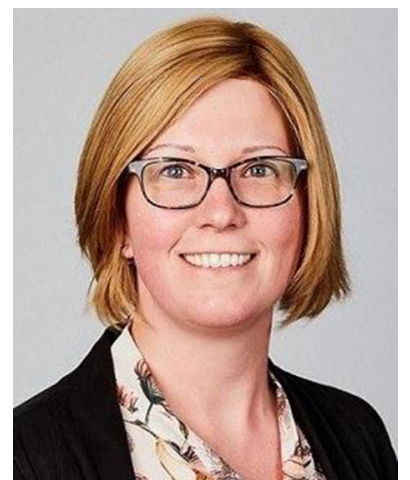

Debby Mangelings is a member of the Department of Analytical Chemistry, Applied Chemometrics and
Molecular Modelling at the Vrije Universiteit Brussel (VUB) in Belgium. Her research focuses on the use of liquid chromatography, miniaturized separation techniques, and supercritical fluid chromatography for a variety of pharmaceutical applications, such as chiral separations, drug impurity profiling, method transferability, skin permeability determinations, and chromatographic fingerprint development in the context of metabolomics. In 2016, she received the LCGC Emerging Leader in Chromatography Award.

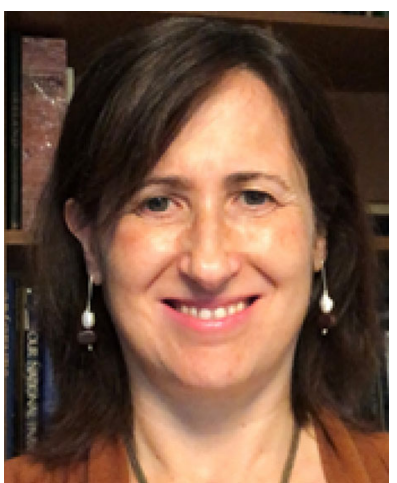

M.-Pilar Marco has a $\mathrm{PhD}$ in pharmacy and is Professor of Research of CSIC. She has developed multidisciplinary scientific activity at the interface of different disciplines, including chemistry, immunochemistry, biochemistry, material sciences, and nanobiotechnology, with the objective of developing a new generation of diagnostic approaches based on the integration of tailored bioreceptors with micro(nano) structures and innovative optical or electrochemical transducer schemes. She is a worldwide expert in the field of antibody technologies, particularly for nonimmunogenic small molecules and the development of innovative diagnostic approaches. Her research has been performed in the context of international collaborations with different research institutions, companies, and other stakeholders and has found application in the biomedical, food safety, and environmental fields. She has co-authored more than 200 articles in international scientific journals of high-impact factor and has filed several patents, some of them under exploitation. Nowadays, she is Coordinator of the Nanomedicine Research Program of the CIBER-BBN (Networking Biomedical Research Center for Bioengineering, Biomaterials and Nanomedicine) and Vice Director of IQAC (Institute for Advanced Chemistry of Catalonia). 


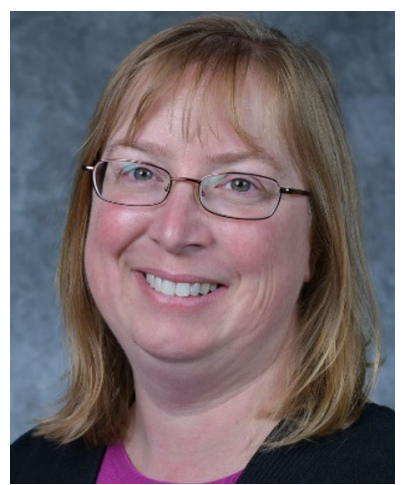

Shelley D. Minteer is the Dale and Susan Poulter Endowed Chair in Biological Chemistry and Associate Chair of Chemistry at the University of Utah. She is also Director of the National Science Foundation Center for Synthetic Organic Electrochemistry. She has diverse interests in fundamental and applied electrochemistry, ranging from biosensors to redox flow batteries and electrosynthesis.

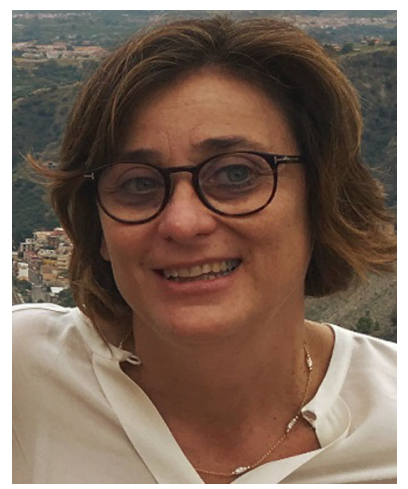

Maria Minunni is Full Professor of Analytical Chemistry, Department of Chemistry "Ugo Schiff", University of Florence, Italy. Her research activity has been mainly focused on the development of bioanalytical assays, in particular, (bio)sensing-based approaches. She is mainly interested in affinity sensing, biomimetic receptors (aptamers, molecularly imprinted polymers), and nanophotonics for a wide range of analytical applications, from molecular diagnostics to food, antidoping analysis, and drug discovery.

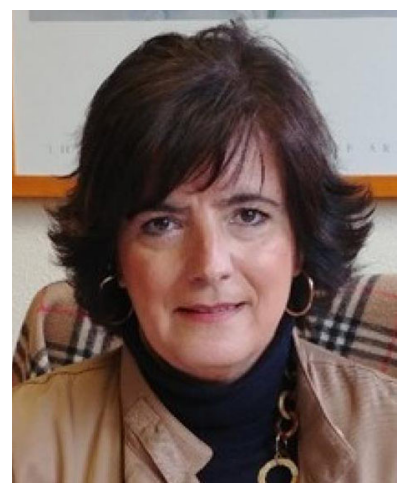

Maria Montes-Bayón is Full Professor of Analytical Chemistry at the University of Oviedo (Spain) and member of the "Mass Spectrometry and Biomedical Analysis Group". She has published more than 140 peer-review publications and has given numerous invited talks. Her research interests are related to the development of analytical strategies for biomedical research, including the analysis of biomarkers in individual cells and the study of the fate of nanostructured materials (encapsulated metallodrugs and nanoparticles) by ICPMS.

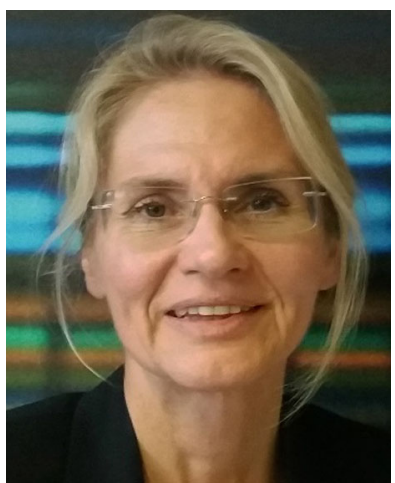

Gertrud E. Morlock holds the Chair of Food Science at the Justus Liebig University Giessen in Germany. She is also Director of the TransMIT Center for Effect-Directed Analysis. She is a leader in the areas of open-source-based miniaturization of planar chromatography, automation of mass spectrometric interfaces, and effect-directed analysis. She hyphenates separation science with biological/enzymatic assays and multi-imaging to discover/identify bioactive unknown unknowns that explain why our food is our medicine. She was awarded the Kurt-Täufel-Preis (since 2017, the WernerBaltes-Preis) des Jungen Wissenschaftlers of the German Society of Food Chemistry and Father of Stevia Award, to name a few.

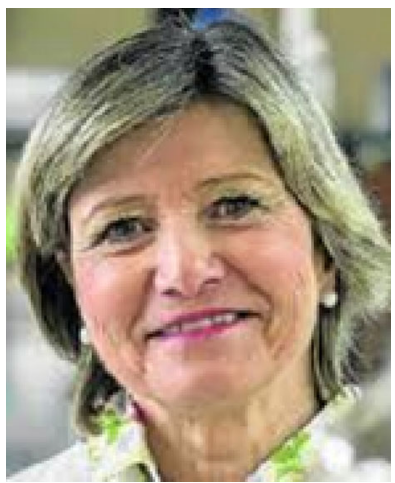

Cristina Nerin is Full Professor of Analytical Chemistry at the Engineering Faculty of the University of Zaragoza (UNIZAR) and Head of the Research group GUIA in the I3A Institute of UNIZAR. Her main research is focused on food-packaging interactions, identification and determination of chemical substances, intentionally and non-intentionally 
added (IAS and NIAS) materials released from packaging, namely, virgin or recycled plastics, paper, board, biopolymers, adhesives, or printing inks, into the packaged product. She is a member of the Board of Directors (BOD) at ILSI (International Life Science Institute) and has been a member of the WG recycling in CEF Panel at EFSA for eight years.

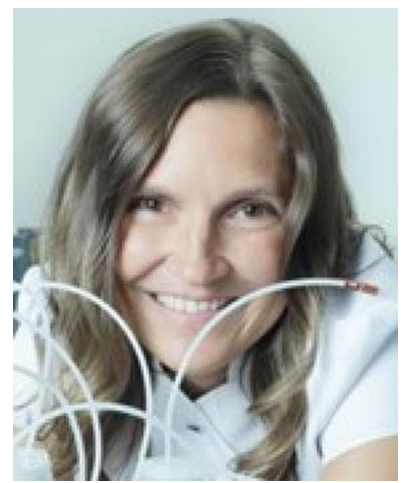

Lucie Nováková is Full Professor in analytical chemistry at the Charles University, Faculty of Pharmacy in Hradec Králové, Czech Republic. Her research is oriented towards separation techniques, namely, ultrahighperformance liquid chromatography, supercritical fluid chromatography, and their coupling to mass spectrometry. She is involved in a wide range of research projects focused on pharmaceutical analysis, doping control, plant analysis, and bioanalytical methods. She authored two books on HPLC theory and practice and nine book chapters. She has published over 110 peer-reviewed scientific articles and review papers, with more than 3350 citations and an h index of 31 . Currently, she is a principal team manager of the STARSS project.

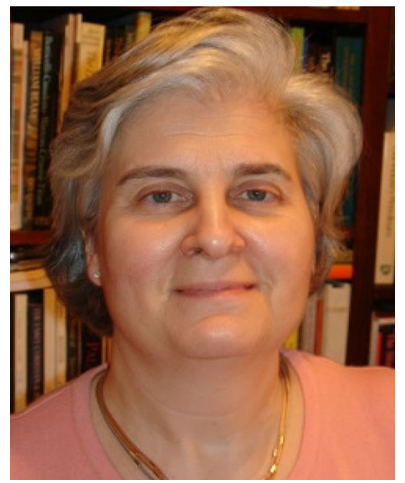

Ana Maria Oliveira-Brett is Head of the Bioelectrochemistry Research Group at the Department of Chemistry, University of Coimbra, and Director of the Electroanalysis and Corrosion Laboratory, at Instituto Pedro Nunes, Coimbra, Portugal. Her research is in the areas of bioelectrochemistry, study of electron-transfer reactions of compounds of biological interest and bioelectroanalysis, development of enzymes and DNA-electrochemical biosensors, and redox mechanisms of amyloid-beta peptides relevant for Alzheimer's disease.

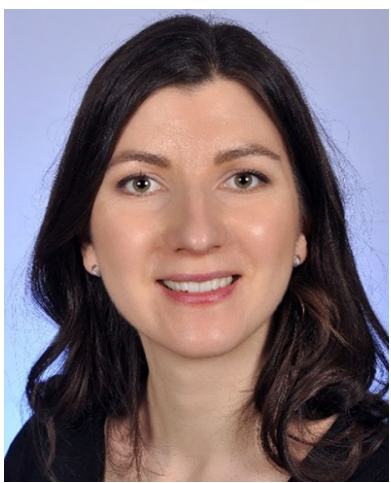

Susanne Pahlow is currently employed as a postdoctoral researcher in the biophotonics group of Prof. Dr. Jürgen Popp at the Leibniz Institute of Photonic Technology (IPHT) and Institute of Physical Chemistry of the Friedrich Schiller University in Jena. She has been working on the development of spectroscopic and molecular biological detection schemes for pathogens for several years. Her main research interests are sample preparation strategies, investigating new capture probes for microorganisms, and surface modification and characterization techniques.

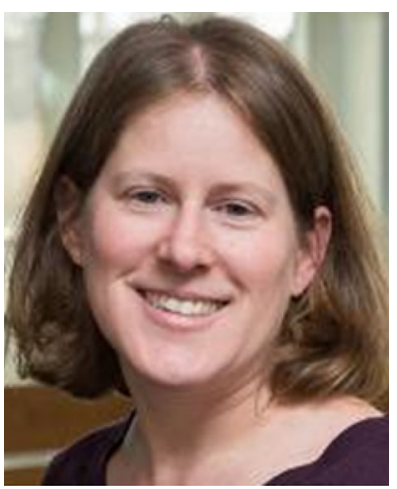

Christine Payne is the Mary Milus Yoh and Harold L. Yoh, Jr. Associate Professor of Mechanical Engineering and Materials Science at Duke University. Her research focuses on understanding how cells interact with nanomaterials. Her lab uses advanced fluorescence microscopy approaches to image this interaction and lab automation to increase throughput and reproducibility. She has received many honors, including an NIH Director's New Innovator Award in 2009 and a DARPA Young Faculty Award in 2011. She earned a B.S. in chemistry from the University of Chicago (1998) and a Ph.D. in chemistry from the University of California, Berkeley (2003). She spent 2003-2006 as an NIH NRSA Postdoctoral Fellow at Harvard University. She joined the faculty of the School of Chemistry and Biochemistry at Georgia Tech in 2007 and moved to Duke University in 2018. 


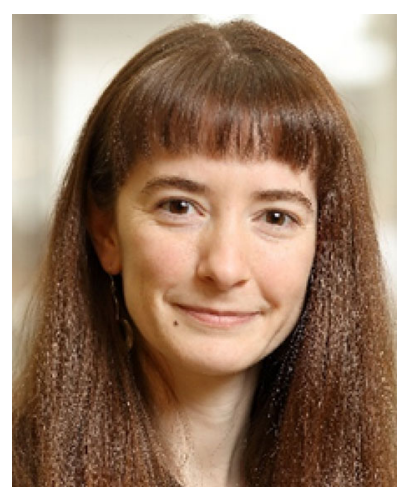

Rebecca Pompano is Assistant Professor in the Departments of Chemistry and Biomedical Engineering at the University of Virginia, and a member of the Beirne B. Carter Center for Immunology Research. She completed a BS at the University of Richmond in 2005 , a PhD in chemistry in 2011 at the University of Chicago, and postdoctoral studies in the University of Chicago Department of Surgery. Her research interests center on developing cutting-edge microscale tools and chemical methods to unravel the complexity of the immune response. Current NIH-funded projects include developing hybrids of lymph node tissue and microfluidics to model inflammation and establishing a spatially organized organ-on-chip model of a lymph node. In recognition of her work, she received an Individual Biomedical Research Award from The Hartwell Foundation, the national Starter Grant Award from the Society of Analytical Chemists of Pittsburgh, a Lab-on-a-Chip Emerging Investigator designation, the Rising Star award from the Cellular and Molecular Bioengineering Special Interest Group, and an inaugural University of Virginia Research Excellence Award. In addition to research, she is active in advancing active learning formats in the analytical chemistry classroom, advocating for continued funding for education and biomedical research on Capitol Hill, and in building inclusive environments for STEM research.

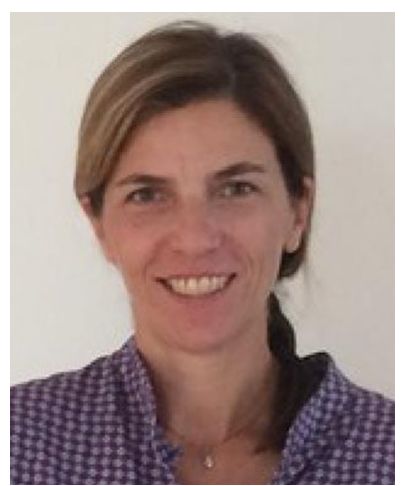

Elefteria Psillakis is Professor in Water Chemistry at the School of Environmental Engineering, Technical University of Crete, Greece. She is Head of the EuChemS-DAC Sample Preparation Task Force and Network. Her research activities focus on understanding the fundamentals and developing new sample preparation methods, and monitoring the environmental fate of organic contaminants.

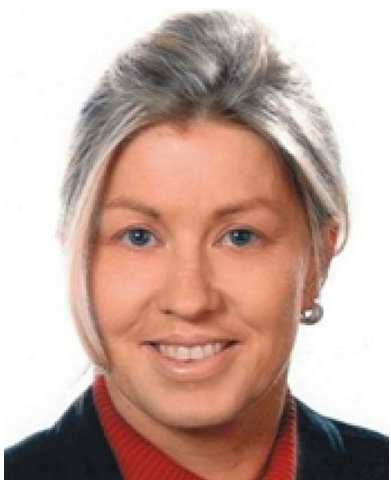

Ute Resch-Genger, $\mathrm{PhD}$, is Head of the Biophotonics division at the Federal Institute for Materials Research and Testing (BAM). She is also co-chair of the steering committee of the Methods and Applications in Fluorescence conference series and member of the editorial advisory board of Bioconjugate Chemistry and the editorial board of the IOP journal Methods and Applications in Fluorescence. Her research interests include photophysics of molecular and nanocrystalline emitters, stimuli-responsive optical probes, signal enhancement, multiplexing, and barcoding strategies, surface group quantification, and concepts for the validation of optical-spectroscopic measurements including fluorescence standards.

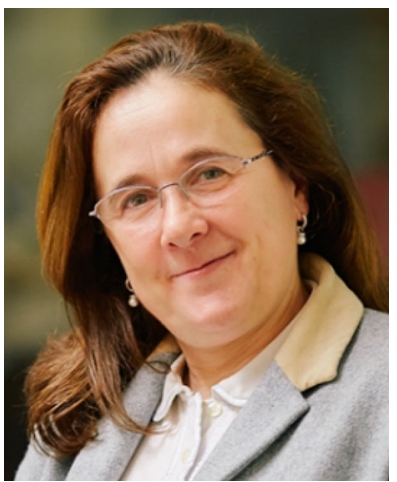

Marina Resmini is Professor of Materials Chemistry at Queen Mary University of London, where she is also Head of the Chemistry Department. She is a Fellow of the Royal Society of Chemistry and Chair of the RSC Inclusion and Diversity Committee; since July 2019, she has also been a member of the RSC Board of Trustees. Her main research interests are focused on the development of novel functional nanomaterials with potential applications at the physical and life sciences interface, in particular, the use of chemistry to confer special properties to materials for applications as drugdelivery systems, catalysts, and sensors. 


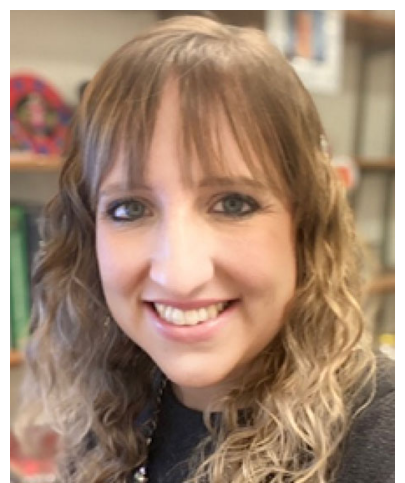

Ashley Ross is Assistant Professor of Chemistry and faculty in the Neuroscience Graduate Program at the University of Cincinnati. Her research focuses on developing both electrochemical and microfluidic tools to study neurochemicalregulated immune signaling in both the brain and immune system.

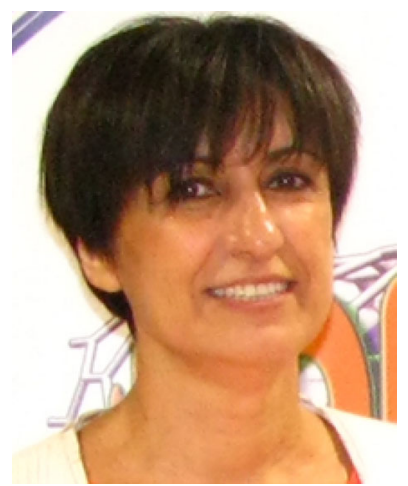

Soledad Rubio Bravo is Head of the Supramolecular Analytical Chemistry Team at the Institute of Fine Chemistry and Nanochemistry at the University of Córdoba, Spain. Her research is focused on the design and synthesis of tailored supramolecular solvents and sorbents for the extraction of bioactive compounds from microalgae and food-processing residues, the treatment of wastewater, and the extraction of organic contaminants in agrifood, biological, and environmental samples prior to their LC-MS analysis.

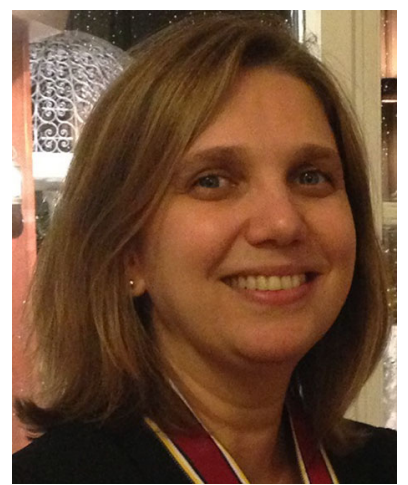

Marcela Segundo is Professor at the Faculty of Pharmacy, University of Porto, and Head of the Analytical Development
Group at REQUIMTE - PT Government Associate Laboratory. She received the FIA award for Science (2016) and she is Secretary of the Division of Analytical Chemistry - EuChemS (2018). She is author or coauthor of $>125$ peer-reviewed publications. Her scientific interests are focused on sample treatment, miniaturization of analytical devices, and hyphenation of flow techniques for high-throughput analysis.

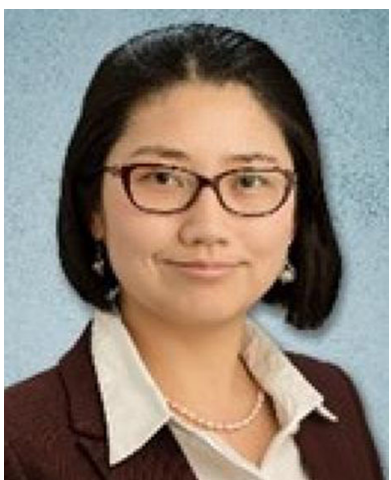

Mei Shen is Assistant Professor of Chemistry and Neuroscience program at the University of Illinois at Urbana-Champaign. She is also faculty for the Beckman Institute for Advanced Science and Technology, Center for Biophysics and Quantitative Biology and Department of Bioengineering. Her research aims to push the limit of electroanalytical chemistry in bioanalysis, especially in studying chemical transmission with nanometer spatial and high temporal resolution. Considering her impact on student scholarship/ intellectual development, and for her innovative approaches to guiding undergraduate research, she received an Award for Excellence in Guiding Undergraduate Research. She is a Scialog Fellow and has received a National Science Foundation (NSF) Career Award to develop multifunctional analytical platforms for neuroanalysis.

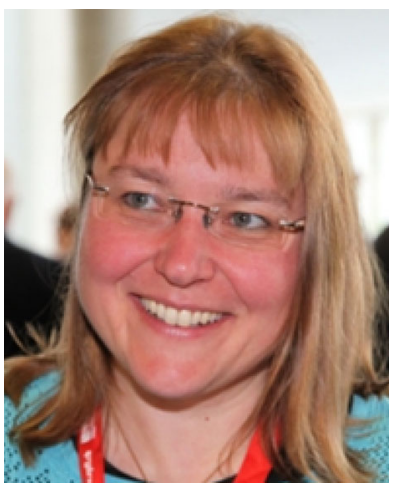

Andrea Sinz is Full Professor and Head of the Department of Pharmaceutical Chemistry and Bioanalytics at the Martin Luther University HalleWittenberg (Germany). She received her degree in 
pharmacy from the University of Tübingen (Germany) in 1993 and obtained her $\mathrm{PhD}$ in pharmaceutical chemistry from the University of Marburg (Germany) in 1997. From 1998 to 2000, she was Post-doctoral Fellow at the National Institutes of Health in Bethesda, MD (USA), where she was introduced to chemical cross-linking and protein mass spectrometry. From 2001 to 2006, she was Head of the junior research group "Protein-Ligand Interaction by Ion Cyclotron Resonance Mass Spectrometry" at the University of Leipzig (Germany), before she accepted her current position at the Martin Luther University Halle-Wittenberg in 2007. Since 2017, she has been President of the German Society for Mass Spectrometry (DGMS). She is an expert in chemical cross-linking and mass spectrometry for studying protein 3D structures and protein interactions. Her research interests are the development of novel analytical strategies and reagents to advance the cross-linking/MS approach. She has authored more than 140 publications and received several awards, such as the Fresenius award of the German Chemical Society (GDCh) in 2019, the Innovation Award in Medicinal/Pharmaceutical Chemistry of the GDCh and the German Pharmaceutical Society (DPhG) in 2006, and the Mattauch-Herzog award of the German Society for Mass Spectrometry (DGMS) in 2004.

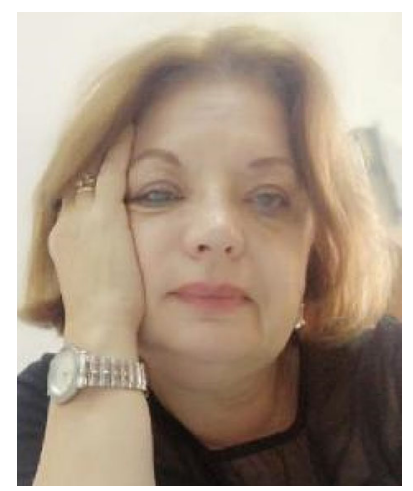

Patricia Smichowski is Head of the Analytical Developments Division at the Atomic Energy Commission of Argentina (CNEA), and she is also Principal Researcher of the National Research Council of Argentina (CONICET). She has led national and international projects and supervised six doctoral theses. She is the author and co-author of over 120 publications, 11 book chapters, and one patent. Her research interests include the development of studies on preconcentration, speciation, and determination of trace elements in a variety of matrices using different analytical techniques and instrumental approaches. She is also interested in physical and chemical characterization of atmospheric aerosols.

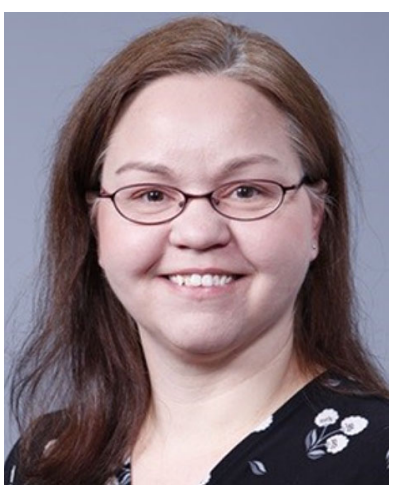

Emily A. Smith is Professor of Chemistry at Iowa State University and Faculty Scientist at US DOE Ames Laboratory. Currently, she is also an American Association for the Advancement of Science, Science and Technology Policy Fellow working on, among other areas, diversity, equity, and inclusion in the physical sciences. She and her research group are developing novel optical spectroscopy instruments for the analysis of plant biomass, mammalian cell membranes, and nanomaterials.

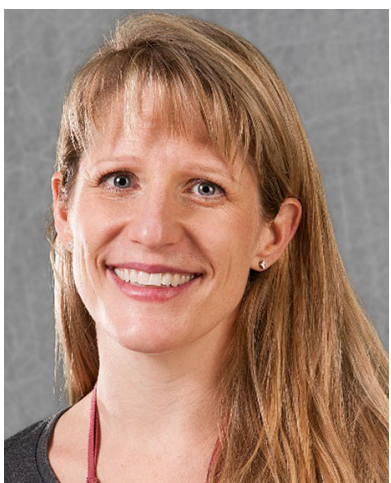

Leslie Sombers is Professor of Chemistry and University Faculty Scholar at North Carolina State University, where she is also graduate faculty in the Department of Biomedical Engineering and a member of the Comparative Medicine Institute. She has won an NSF CAREER Award and an NIH Early Career Award in the Chemistry of Drug Abuse and Addiction. Her research program lies at the interface of neuroscience, engineering, and analytical chemistry. The Sombers lab is particularly focused on the development of new electroanalytical tools for the real-time monitoring of neurochemicals in systems ranging from subcellular domains to discrete brain regions in awake and behaving animals. 


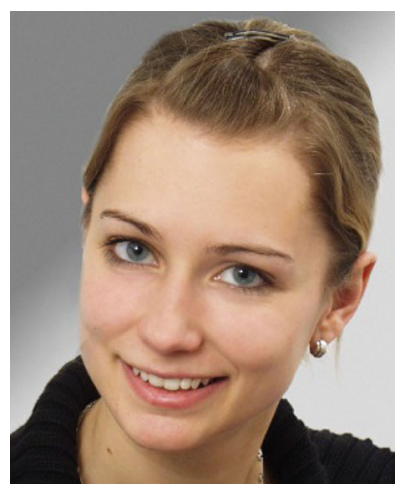

Andrea E. Steuer is Deputy Head of the Department of Forensic Pharmacology and Toxicology of the Zurich Institute of Forensic Medicine at the University of Zurich. She has been working for several years in the area of clinical and forensic toxicology doing both service work (expert reports for police and state attorneys) and research. Her main research focus is on different aspects of drugs of abuse and/or postmortem analysis using different hyphenated mass spectrometry techniques, from method development and metabolism studies to metabolome investigations.

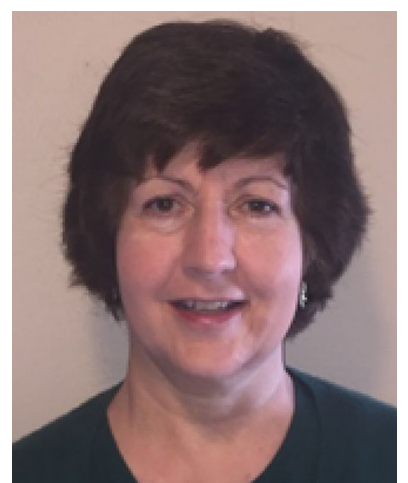

Sherri B. Turnipseed joined the US Food and Drug Administration in 1993 as a research chemist in the Animal Drugs Research Center. Her primary responsibility has been to develop and validate methods for animal drug residues in complex food matrices using mass spectrometry. For the past several years, her research has focused on the use of highresolution mass spectrometry to expand residue monitoring capabilities to additional analytes.

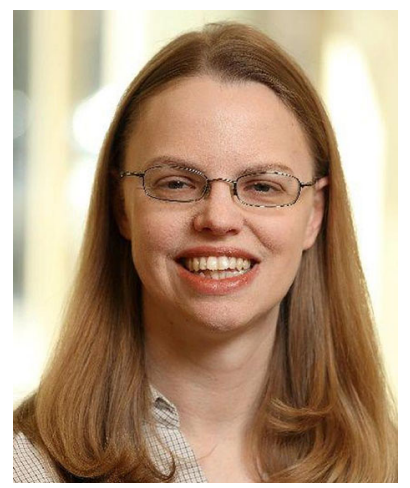

B. Jill Venton is Professor and Chair of the Department of Chemistry at University of Virginia. She is also affiliated with the Neuroscience Graduate Program and Brain Institute. Her research interests are in developing new electrochemical and separations techniques for measurements of neurotransmitters in tissue.

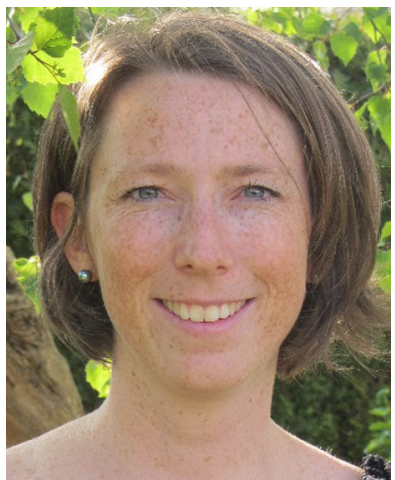

Caroline West is Associate Professor in Analytical Chemistry at the University of Orleans, France. She is also a junior member of the French University Institute, a service of the Ministry of Higher education distinguishing a small number of university professors for excellent research. Her main scientific interests lie in fundamentals of chromatographic selectivity, in both achiral and chiral modes, mainly in SFC, but also in HPLC, and applications to compounds of pharmaceutical interest.

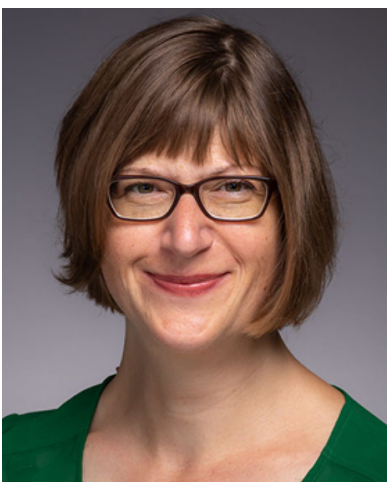

Rebecca Whelan is Associate Professor of Chemistry and Biochemistry at the University of Notre Dame, where she is also affiliated with the Harper Cancer Research Institute and the Center for Advanced Diagnostics \& Therapeutics. She previously served as Associate Professor and Chair of the Department of Chemistry and Biochemistry at Oberlin College. Her research seeks to develop new bioanalytical tools to solve challenging problems in human health and draws on small-scale separations, proteomics, functional nucleic acid selection, bioinformatics, and nanotechnology. 


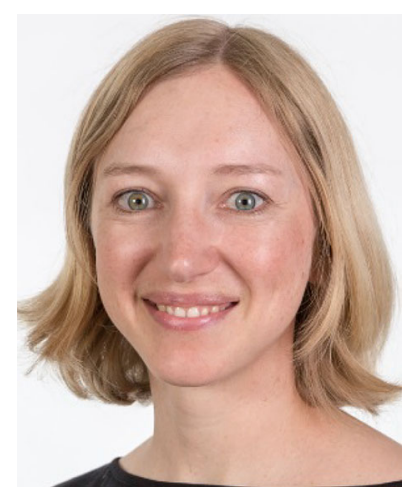

Therese Wohlschlager is Postdoctoral Researcher at the University of Salzburg, Austria. As a member of the Christian Doppler Laboratory for Biosimilar Characterization, she focuses on the development of HPLC and mass spectrometry based approaches for the characterization of therapeutic proteins. For her work in the field of glycoprotein analysis, she received the Life Science Research Award Austria 2018 and the Fritz-Feigl-Award 2019.

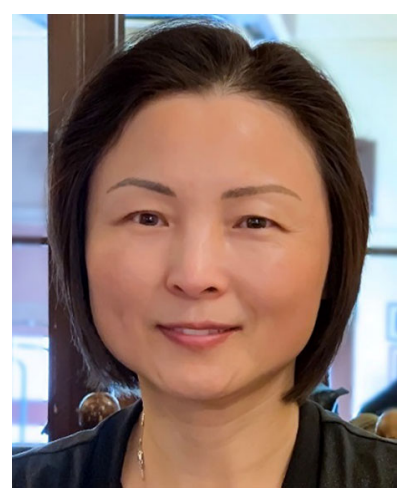

Lu Yang is Senior Research Officer at the National Research Council of Canada (NRC, Ottawa, Canada), leading research in applications of ICP-MS and MC-ICP-MS. The isotopic measurements of mercury, germanium, indium, iridium, osmium, hafnium, and lead from her lab have been adopted by IUPAC as the best available isotopic composition measurements, and the standard atomic weights of mercury, germanium, iridium, and hafnium are based on her lab results. She has published over 130 peer-reviewed publications and a book chapter, with an $h$ index of 33 . She is an editorial board member of JAAS and Spectroscopy.

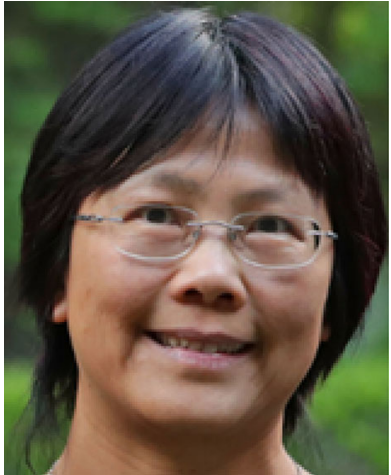

Wenwan Zhong is Professor of Chemistry at the University of California, Riverside. The Zhong group focuses on the development of advanced analytical techniques for the study of epigenetic factors, employing separation tools, biosensing assays, supramolecular chemistry, functional nucleic acids, and nanomaterials. She is also interested in exploration of the nano-bio interface for a better understanding of the biological impacts of nanomaterials. She received the NSF CAREER award in 2011 and UCR Chancellor's Award for Excellence in Undergraduate Research in 2019.

Publisher's note Springer Nature remains neutral with regard to jurisdictional claims in published maps and institutional affiliations. 